\title{
Zur symbolischen Rückeroberung und Politisierung des öffentlichen Raums ${ }^{1}$ Eine Analyse der Raumpolitiken des movimiento 15-M
}

\section{Einleitung}

Tahrir - Syntagma - Puerta del Sol: drei symbolisch bedeutsame und miteinander verknüpfte räumliche Referenzpunkte der internationalen Protestbewegungen des Jahres 2011 - drei Repräsentationen der aufbrechenden Risse im Kapitalismus - drei Beispiele für acts of citizenship ${ }^{2}$, die weiterführende Überlegungen zu den gegenwärtigen Entwicklungen eines bedeutsamen politikwissenschaftlichen Begriffs in einer von vielfältigen identitätspolitischen Zuschreibungen geprägten und neuartigen sozialen Bewegungen aufgerüttelten Welt erlauben. Trotz aller Differenzen zwischen dem arabischen Frühling und den südeuropäischen Protestbewegungen lassen sich drei gemeinsame Aspekte festhalten: Erstens reagieren sie auf die tiefgreifenden Brüche im Finanzkapitalismus und wenden sich gegen vermeintlich alternativlose Lösungen seiner Krise, die pikanterweise die ökonomischen und politischen Eliten begünstigen, während von den Anpassungsmaßnahmen in erster Linie die gesellschaftlichen Mehrheiten betroffen sind. Zweitens haben sie sozialpolitische Forderungen, die eine andere Art der Krisenbewältigung einklagen, mit dem Verlangen nach neuen Formen der demokratischen Willensfindung im Zeitalter delokalisierter politischer Kommunikation verknüpft ${ }^{3}$. Drittens konstituieren sie neue Ausdrucksformen

1 Dieser Beitrag entstand im Rahmen des von der Europäischen Kommission geförderten Forschungsprojekts NEOLIBERAL_CITI: Re-framing urban neoliberalism and neo-liberal citizenship-Enactments of resistance and practices of protest (PERG-08-GA-2010-277115).

2 Da dieser Text an konzeptionelle Debatten der angelsächsischen Citizenship Studies anknüpft, wird nachfolgend überwiegend der englische Begriff citizenship verwendet. Damit wird die in deutscher Sprache anders gelagerte, stärker auf die Rolle des Staates fixierte und im Kontext des movimiento 15-M eher hinderliche Bedeutungszuschreibung von Termini wie Bürgerschaft, Staatsbürgerschaft oder Staatsangehörigkeit umgangen.

3 Vgl. hierzu den Beitrag von Espinar/Abellán in diesem Heft sowie Espinar 2012. 
politischen Protests, in denen sich die Mobilisierung gleichzeitig in 'virtuellen' sozialen Netzwerken und an konkreten, symbolisch bedeutsamen städtischen Orten kristallisiert ${ }^{4}$. Shahyar (2011) zufolge nähren sich die Revolten zudem aus der Unzufriedenheit 'avantgardistischer' sozialer Milieus, d.h. von erwerbslosen jungen Menschen mit weiterführenden Bildungsabschlüssen ${ }^{5}$. Genau dieses neue 'ökonomische Prekariat' verfügt aber nicht nur über ein ausgeprägtes Sozialkapital und Organisationstalent, sondern besitzt aufgrund fehlender Perspektiven auf dem Arbeitsmarkt auch Motive und zeitliche Ressourcen zur Begründung neuer sozialer Bewegungen.

In Anbetracht eines neuartigen Panoramas sozialer Auseinandersetzungen, das sich seit Beginn des Jahres 2011 zunehmend globalisierte, stellt sich nunmehr die Frage, wie eine kritische Sozialwissenschaft im Schnittfeld von Stadtgeographie und Politischer Theorie zum Verständnis und zur Konzeptualisierung des gegenwärtigen Widerstreits beitragen kann. In Bezug auf das movimiento 15-M ${ }^{6}$ kann dabei in erster Linie das sprichwörtliche 'Hereinbrechen' emanzipatorischer Kämpfe in die spanische Öffentlichkeit konstatiert werden, welche sich anhand neuer politics of place (Amin 2004; Massey 2004) manifestieren. In dieser relationalen Konzeption von place sind Orte Gegenstand machtvoller Diskurse und spielen für politische Bewegungen eine grundlegende Rolle (Leitner et al. 2008). Im konkreten Fall wird die soziale Bewegung durch die symbolische Verknüpfung der Auseinandersetzungen mit dem Signifikanten des Platzes der Puerta del So $\Gamma$ inszeniert; die Bedeutungen, die durch diese Verknüpfung entstehen, werden im weiteren Verlauf der Proteste auf die zahllosen öffentlichen Plätze und Straßen, auf denen Stadtteilversammlungen und andere Zusammenkünfte organisiert werden, übertragen. Das movimiento $15-M$ verkörpert einen radikalen Wandel der Öffentlichkeit. Dieser erfolgt durch die Rückeroberung und Politisierung des öffentlichen Raums - eines Raums, der sich im Zuge der neoliberalen Pro-

4 Zur Rolle der sozialen Netzwerke in der Entwicklung des movimiento 15-M vgl. Hughes 2011 und Abellán 2012.

5 Während die allgemeine Jugendarbeitslosigkeit in Spanien mittlerweile die Marke von 48 Prozent erreicht, beträgt sie selbst unter jungen Universitätsabsolventen erschreckende 36 Prozent, bei einem landesweiten Durchschnitt von über 23 Prozent der arbeitsfähigen Bevölkerung.

6 In diesem Artikel wird die Bezeichnung movimiento 15-M verwendet, obwohl der Begriff gleichzeitig eine medial konstruierte Homogenisierung eines vielschichtigen Phänomens darstellt. Die Stärke der 'Marke' 15- $M$ liegt jedoch darin, dass mit diesem Signifikanten gleichzeitig alles und nichts bezeichnet werden kann und so eine identitätspolitische Gruppenkonstuktion ermöglicht wird.

7 Die Puerta del Sol ist der zentrale städtische Platz in Madrid, Sitz der Regionalregierung und der Ort, an dem im Mai und Juni 2011 die Aktivisten des 15. Mai campierten. 
duktion des Städtischen zusehends zu einer Restkategorie des Immobiliensektors sowie zum physischen Ort 'kontrollierter' und gewinnorientierter Tätigkeiten entwickelt hatte.

Im Folgenden werden zunächst am Beispiel der spanischen Städte die vielfältigen Logiken der Raumproduktion im neoliberalen Zeitalter sowie die daraus resultierende Transformation des öffentlichen Raums verhandelt. Darauf aufbauend wird eine Interpretation der gegenhegemonialen Raumpolitiken der Bewegung des 15. Mai vorgenommen, die sich auf Engin Isins $(2008,2009)$ Konzeptualisierung von acts of citizenship stützt und die Schaffung einer neuen Form der Öffentlichkeit und des öffentlichen Raums durch bewusste Gehorsamsverweigerung, ja mutwillige Überschreitung von Normen und Gesetzen hervorhebt. Die in vielen spanischen Städten verwirklichten mehrwöchigen Zeltlager des Frühsommers 2011, im öffentlichen Raum abgehaltene Versammlungen sowie die seit Oktober 2011 vollzogenen Hausbesetzungen können aus dieser Perspektive als neu geschaffene spaces of citizenship analysiert werden, in denen sich auf die Rückeroberung des öffentlichen Raums und die Rekonstruktion einer politischen und politisierten Öffentlichkeit abzielende Raumpolitiken konsolidieren und eine andere Art der Politik eingefordert wird.

\section{Zur Raumproduktion im neoliberalen Zeitalter - Öffentlichkeit und öffentlicher Raum in der Defensive}

Ein zentraler Aspekt der Stadtpolitiken im neoliberalen Zeitalter besteht in der Durchsetzung und Diffusion neuer Regulationen, Programme und Mikropolitiken, die aus einer ökonomischen Perspektive die Stadt 'in Wert' setzen und sie so rekonfigurieren. In grundsätzlicher Hinsicht lassen sich die Konsequenzen dieser Politiken unter dem Stichwort einer primär an privatwirtschaftlichen Interessen ausgerichteten Verwaltung sowie der damit verbundenen zunehmenden Vermarktung der Städte fassen, unter die auch die weitreichende Deregulierung und Privatisierung städtischer Dienstleistungen fällt (Janoschka 2011). Diese neoliberale Strukturreform des städtischen Raums erfolgt in aller Regel zu Ungunsten ohnehin benachteiligter Bevölkerungsgruppen und treibt deren Expulsion voran (Smith 1996), wobei der Rekurs auf die Marktlogik die Reproduktion sozialer Strukturen sowie der in ihnen zum Ausdruck kommenden Raumnutzungen befördert.

In vielfältigen Debatten ist darauf hingewiesen worden, dass der öffentliche Raum nicht nur ein geographisches, architektonisches und damit genuin städtisches Konzept darstellt, sondern eng mit den Strukturationsprinzipien der politischen Öffentlichkeit verbunden ist (Habermas 1990; Benhabib 1991; 
Howell 1993). Als Ort der freien Meinungsäußerung und der politischen Deliberation, für alle Bürger zugänglich, hat der öffentliche Raum aber spätestens im Zuge der neoliberalen Restrukturierung der Städte ausgedient. Unter der liberalen Vorstellung eines öffentlichen Raums versteckt sich nurmehr eine scheinbare Gleichheit in Bezug auf die Nutzungs- und Aneignungsmöglichkeiten durch unterschiedliche soziale Gruppen. Delgado (2011) zufolge hat sich der öffentliche Raum in einen politisch sinnentleerten Ort verwandelt, der nur noch innerhalb des von Investoren und öffentlichen Verwaltungen vorgegebenen Rahmens angeeignet werden kann; ein Rahmen, in dem die politische Deliberation ebenso wie der Ausdruck von Differenz nicht vorgesehen ist. In diesem Sinne haben sich öffentliche Räume zusehends in aseptische Orte verwandelt, in denen Präventionsmaßnahmen und Überwachungsstrategien angewendet und unter Berufung auf Sicherheit soziale Kontrollmechanismen organisiert werden (Wehrheim 2002). Traditionell verankerte und alternative Nutzungsmuster werden so unterbunden, und die mit der Disziplinierung gepaarte Ökonomisierung leistet weiteren Transformationen wie der Gentrifizierung Vorschub (Low, Smith 2006; Lees et al. 2008). Aus einer anderen Perspektive kann dies auch als Implementierung von Techniken der Klassenherrschaft interpretiert werden (Brenner et al. 2010; Garnier 2011).

Derartige Prozesse sind mittlerweile dermaßen normalisiert, dass sie außerhalb kritischer sozialwissenschaftlicher Analysen kaum noch wahrgenommen werden. Doch die Bürger auf den Terrassen und die auf Bänken, diejenigen, die in die Boutiquen eintreten und dort einkaufen, und diejenigen, die diese Geschäfte kaum wahrnehmen, repräsentieren zwei diametral entgegengesetzte Modelle von citizenship. Die Kontrollarchitektur reguliert die möglichen Begegnungen, verhindert diese, beherrscht die Interaktionen und diszipliniert die Körper. Gleichzeitig ist es zunehmend der Bürger selbst, der unbewusst als Polizist eingesetzt wird, eine Mikroüberwachung praktiziert und so hilft, die städtischen Räume aufzuwerten. Diese Logik ist es, die im Mittelpunkt des neoliberalen städtischen Projekts steht: distinktiver Konsum als Dreh- und Angelpunkt der Reproduktion von hedonistisch und narzisstisch geprägten sozialen Feldern. Auch die Raumproduktion bleibt von dieser Logik nicht unberührt, im Gegenteil, sie ist materieller Ausdruck der sozialen Reproduktion und Inszenierung an spezifischen Orten. In dem Versuch, Geselligkeit in architektonische Formen $\mathrm{zu}$ fassen, werden die Orte der Begegnung vorgestaltet, in einem Versuch, das Unvorhersehbare zu verwalten. Diese Gestaltung ist im Sinne Foucaults (1990) als ein Bündel von Technologien zu begreifen: Technologien der Produktion, mit deren Hilfe Dinge verändert und manipuliert werden; Technologien der Zeichensysteme, welche die Verwendung von Zeichen, Symbolen und Sinngehalt ermöglichen; Technologien der Macht, mit denen das Verhalten von Individuen 
konditioniert und in Richtung bestimmter Ziele gesteuert wird; Technologien des Individuums, die Bürgern bestimmte Gedanken und Verhaltensweisen zur Erfüllung bestimmter Zielsetzungen vorgeben. Diese Kategorisierungen eröffnen unterschiedliche Möglichkeitsräume: die Unterwerfung unter die Regierung und gelenktes Verhalten; oder die Ablehnung, Verweigerung und Rebellion. Aus der zweiten Alternative entwickelt Foucault ein Verständnis, demzufolge diejenigen, die sich der Disziplinierung verweigern und sich gegen diese auflehnen, sich nicht auf die Kritik von Institutionen beschränken sollen: Es handelt sich um die herrschende politische Rationalität, die kritisch hinterfragt und auf den Prüfstand gestellt werden soll.

\section{Macht und Gegenmacht: Acts of citizenship, spaces of citizenship}

Die zuvor geschilderte, der Raumproduktion inhärente Beziehung, Macht zu beherbergen und zum Ausdruck zu bringen, wird durch den Verweis auf die symbolischen Dimensionen von Orten oder die politics of place deutlicher. Der Prozess der Herstellung von Orten ist ein interessanter Analyserahmen, in dem die Aufdeckung besonderer Konstellationen von Bedeutungen, Ästhetik, Werten und naturalisierter sozialer Vorschriften erfolgen kann. Politische Bewegungen streben eine Veränderung der Bedeutungszuschreibungen symbolisch wichtiger Orte an und stimulieren so die Produktion neuer, strategisch aufgeladener Orte, die ihren eigenen Projektionen, Prioritäten und Vorstellungen entsprechen (Leitner et al. 2008). In Bezug auf die Bewegung des 15. Mai lässt sich zeigen, wie die politics of place zur symbolischen Rückeroberung von Räumen und Orten verwendet werden. So wurde die Puerta del Sol, ein touristisch und kommerziell geprägter Ort in der Mitte der zentralen Einkaufsstraßen der spanischen Hauptstadt, in das politische Zentrum der Bewegung verwandelt und so die deliberative demokratische Funktion von Öffentlichkeit im öffentlichen Raum zurückerobert.

Diese Rückeroberung und Politisierung des öffentlichen Raums, die nicht nur für die Puerta del Sol, sondern auch eine Vielzahl anderer Plätze und Straßen, die zur Bühne von Demonstrationen und Versammlungen wurden, charakteristisch ist, kann nun konzeptionell aus der Perspektive der Konstruktionsprinzipien von citizenship betrachtet werden. Dabei knüpfen wir an Debatten der politischen Philosophie an, in denen die Veränderungen der Staatsbürgerschaft [citizenship] in globalisierten Gesellschaften verhandelt werden. Unter anderem werden darin die tradierten Vorstellungen über die Rollenverteilung in Staat und Gesellschaft (Marshall 1950; Arendt 1958) kritisiert und gleichzeitig die praktischen und alltagsweltlichen Dimensionen einer angewandten und sozial konstruierten citi- 
zenship betont (Isin/Wood 1999; Ong 2006). Diese Sichtweise negiert nicht die Rechte und Pflichten von Bürgern - sie erweitert die theoretischen Perspektiven, um Antworten auf die veränderten Bedingungen sozialer und politischer Zugehörigkeit in einer transnational organisierten Weltgesellschaft zu finden (Barnett 2003; Low 2004). Angereichert um kulturelle, ökonomische und symbolische Praktiken kann ein in Zeit und Raum formbares Konzept entworfen werden, auf das strategisch rekurriert wird, um die Forderungen sozialer Gruppen zu stützen, anzuerkennen und auszuweiten. In Bezug auf die Bewegung des 15. Mai wollen wir diese Perspektive schärfen und an ihr eine Art von citizenship diskutieren, die partizipativ, fließend, als Ausdruck identitätspolitischer Positionierungen gedacht wird und sich als performativer Akt in der gesellschaftlichen Praxis artikuliert. So gedacht, ist citizenship ein Objekt der permanenten Auseinandersetzung und Aushandlung, eine Praxis und eine Fähigkeit zu agieren (Rose 2001).

Wenn die Akte der Rebellion des movimiento 15- $M$ aus dieser Perspektive gedacht werden, so lässt sich eine theoretisch fundierte Antwort entwerfen, in der sich die Debatten zu Raum und Räumlichkeit einfügen. So kann insbesondere die konfliktive Perspektive von citizenship geschärft werden, und zwar unter Berücksichtigung der politischen Konflikte, die seit der Besetzung der Puerta del Sol enstanden sind und die neue Akteure, Orte und Skalen von citizenship geschaffen haben (Isin 2009). In den politischen Akten des movimiento 15-M konstituieren sich neue Akteure als Subjekte, die klare Forderungen aussprechen, und zwar unter Referenz auf symbolisch aufgeladene Orte. Allmählich weicht so die Machtrelation zwischen Bürger und Staat auf; sie wird neu entworfen, und zwar anhand einer Art von citizenship, die sich auf die spezifischen Akte konzentriert, in denen neue Ansprüche erhoben werden (Isin 2008). Diese acts of citizenship basieren als konstitutiver Prozess einer neuen Bürgerschaft primär auf den erwähnten Brüchen, Zerrüttungen und Abweichungen, wobei die Erkundung der Formen, anhand derer citizenship als relationales Konzept entwickelt wird, in den Mittelpunkt der Betrachtungen gerückt wird (Aradau et al. 2010). Solch eine radikale Relationalität impliziert, dass die Ausdrucksformen (Akte), die Orte und die Skalen der Identifikation von citizenship substanziell anders als die hegemoniale Organisation der Welt geartet sind. Die Bürger werden demnach als sich im Konflikt, in den politischen Prozessen und sinnstiftenden Akten erst konstituierende Subjekte betrachtet. Hierbei wird der Fokus auf die mit den Routinen und alltäglichen Praktiken brechende Akte gelegt.

Unter Bezugnahme auf Tamayo (2006) lässt sich darauf aufbauend argumentieren, dass sich die Kämpfe zur Durchsetzung neuer Rechte in den grundlegenden Motor des politischen und sozialen Wandels verwandelt haben und es die symbolischen Kampfhandlungen sind, in denen sich die Subjekte in das verwandeln, was Isin als activist citizen bezeichnet. In den umkämpften Aneignungen 
und Rückeroberungen des öffentlichen Raums entstehen neue spaces of citizenship - Räume, die gleichzeitig objektive (physische) und subjektive (gemeinschaftliche) Dimensionen beinhalten. So wird dem Konzept der citizenship eine Komplexität verliehen, in der gleichzeitig auf Dimensionen von Räumlichkeit und Macht verwiesen wird. Wie in den nachfolgenden Betrachtungen zum movimiento 15-M nun weiter ausgeführt wird, kann so eine relationale räumliche Komponente in die Umdeutung von citizenship als Resultat sozialer Kämpfe eingeführt werden.

\section{Movimiento 15-M und der öffentliche Raum: Acts of Citizenship und die Umdeutung von Raumpolitiken}

Im Anschluss an die namensgebende Demonstration des 15. Mai waren es eine handvoll Aktivisten, die an der Puerta del Sol in improvisierten Zelten übernachteten - erst die einen Tag später erfolgende Räumung des Platzes motivierte die Solidarität und Empörung und stellt die Grundlage der spontanen Eroberung des Platzes durch die Bevölkerung dar. In einer raschen und konzertierten Aktion forderten die Aktivisten unterschiedlicher sozialer Bewegungen nun im öffentlichen Raum dessen politische Dimension ein und eroberten Räume der Begegnung, Debatten, Proteste, welche in die Schaffung neuer Subjektivitäten münden. In kürzester Zeit wurde eine beispiellose logistische Aktion vollbracht, in der die Grundlage einer 'Stadt' des Widerstands errichtet wurde, mit thematischen Kommissionen, die politische, aber auch spielerische Aktivitäten organisierten. Dieser erste Moment begründete das movimiento 15- $M$ und öffnete den Weg zu einer Neudefinierung und Rückeroberung des öffentlichen Raums und einer politischen Öffentlichkeit, die hier in zwei Dimensionen analysiert werden soll: einerseits die Umdeutung der im öffentlichen Raum normalisierten Praktiken sowie die Rückeroberung des öffentlichen Raums durch die politische Öffentlichkeit und andererseits die Schaffung neuer öffentlicher Räume zur Reartikulierung des Gemeinsinns.

Die Rückeroberung des öffentlichen Raums ist anhand des Protestcamps sowie der ab Mitte Juni einsetzenden, auf öffentlichen Plätzen abgehaltenen Stadtteilversammlungen nachvollziehbar. Die Bewegung des 15. Mai zeugt von dem subversiven, latent vorhandenen Widerstand gegen die gesellschaftlichen Disziplinierungsversuche. Sie verwandelt Straßen und Plätze in 'echte' öffentliche Räume, in denen nicht nur Politik, sondern auch das übrige soziale Leben stattfindet und gegessen, getrunken, gefeiert, diskutiert, gespielt und gelesen wird und neue Formen von Gemeinschaft erprobt werden. Dabei spielt die Regierungspolitik der Bewegung mit zweifelhaften Entscheidungen in die Hand. Jeder Versuch, die Bewegung zu stigmatisieren, zu illegalisieren oder 
Demonstrationen zu verbieten, multipliziert in einer an offene Revolte gegen die disziplinierende Staatsgewalt grenzenden Empörung die Zahl derer, die das Protestcamp frequentieren und mit der Bewegung sympathisieren. Diese Akte der Rebellion erzeugen neue Identitäten von Kollektivsubjekten und begründen den gesellschaftlich bis heute relevanten Mythos des movimiento 15-M. Nachdem diese Praktiken über Wochen an der Puerta del Sol sowie auf den umliegenden Plätzen und Straßen, die ebenfalls in Versammlungsorte verwandelt und unter dem Bruch konventioneller Raum-Zeit-Schemata eingeübt worden sind, erfolgte in einem zweiten Schritt dann die Eroberung der Stadtteile. In einer zentrifugalen Bewegung wurden die Logiken des bis dahin zentralisierten, aber in Zeit und Raum nicht dauerhaft möglichen Protestcamps auf die gesamte Stadtregion ausgeweitet und eine Politisierung des öffentlichen Raums in traditionellen Stadtvierteln, in unmenschlichen Neubausiedlungen der urbanen Peripherie, in suburbanen Einfamilienhaussiedlungen und entfernten Dörfern erreicht. Die Vertiefung der horizontalen Demokratie in den Stadtteilen, nach Prinzipien, die auf dem Platz der Puerta del Sol kollektiv erarbeitet und erprobt worden sind, entspricht der Artikulation neuer Dimensionen der acts of citizenship sowie der Schaffung neuer Akteure mit Referenz auf ortsbezogene Politiken. Auf diesem Wege erfolgt eine Territorialisierung kollektiver Identitäten, die sich in räumlich eingebetteten Forderungen nach lokaler Demokratie niederschlagen. So wurden konkrete stadtpolitische Ansprüche an die lokalen Lebensbedingungen und den kollektiven Konsum in eine soziale Bewegung integriert, die eine neuartige Konzeption von Öffentlichkeit in alltäglichen Denk- und Handlungsschemata zum Ausdruck bringt. Vor dem Hintergrund, dass die gebaute Stadt ein Ausdruck konfliktiver Interessen von Akteuren mit antagonistischen sozialen Positionen ist (Sequera 2009, 2011), stellen die neuen Formen basisorientierter Debatten bei im öffentlichen Raum stattfindenden Stadtteilversammlungen eine Erweiterung der demokratischen Rechte dar, die erst durch die bewusste Überschreitung gesetzter Grenzen, d.h., durch acts of citizenship erkämpft wurde. Gleichzeitig sind es die seit Monaten stattfindenden Versammlungen, in denen sich eine zunehmende programmatische Positionierung der Bewegung entwickelt.

Aus den Orten einer eingeübten radikalen Demokratie, die in den Stadtteilversammlungen zum Ausdruck gebracht wird, erwächst eine Reihe weiterer kollektiver Aktionen - uns interessieren hierbei insbesondere diejenigen, die sich gegen eine spezifische Konsequenz der spanischen Wirtschaftskrise richten, nämlich die Zwangsversteigerungen sowie den spekulativen Leerstand von Wohnungen und Häusern. Während allein in der Region Madrid seit Beginn der Krise mehr als 50.000 Zwangsversteigerungen und Wohnungsräumungen durchgesetzt wurden, stehen gleichzeitig über 300.000 Wohnungen leer. Mit dem Verweis auf das 'Recht auf Stadt' sowie das in der spanischen Verfassung 
festgeschriebene 'Recht auf eine angemessene Wohnung' wird ab dem Herbst 2011 eine zunehmende Zahl von Gebäuden besetzt und zu vom kapitalistischen Zwang ‘befreiten' Orten erklärt. Die Besetzungen richten sich in erster Linie gegen die Spekulation mit Wohnraum, weshalb vor allem leerstehende, im Besitz von Banken und Immobilienfirmen befindliche Gebäude ausgewählt werden. Als erstes Objekt wurde in Madrid ein leer stehendes Hotel besetzt, 300 Meter von der Puerta del Sol entfernt und in direkter Nachbarschaft des regionalen Wirtschaftsministeriums. Die medienwirksam inszenierte Besetzung des Hotels erfolgte im Anschluss an die mit 500.000 Demonstranten bis dato massivste Kundgebung am 15. Oktober und markierte einen Wendepunkt der Bewegung. Im Vorfeld der anstehenden Wahlen wurde hier ein Machtvakuum ausgenutzt, um eine neue Strategie des Widerstands zu erproben und zu etablieren. Im Hotel erhielten sukzessive mehr als 100 zwangsenteignete Familien eine temporäre Behausung, und es gelang zudem, eine im Gesetzbuch als Straftat gebrandmarkte Aktivität gesellschaftlich zu legitimieren. Gleichzeitig wurde ein den Witterungsbedingungen im Herbst angemessener Ort zur Durchführung von Versammlungen und Kommissionssitzungen geschaffen und der Bewegung ein neues symbolträchtiges 'Zuhause' verliehen. Im 'Hotel Madrid' lag auch die Keimzelle des neu gegründeten Wohnungsbüros, das von Zwangsenteignungen betroffenen Bürgern als Anlaufstelle dient und diese in der Mehrzahl unpolitischen Individuen auch in Fragen der Selbstorganisation und Vorbereitung neuer Hausbesetzungen schult. Mittels dieser Vorbereitung breiteten sich die Hausbesetzungen seit Ende Oktober landesweit aus, und auch in vielen Stadtteilen und Umlandgemeinden Madrids wurde sukzessive eine ganze Reihe von Gebäuden besetzt, unter anderem leer stehende Markthallen oder Gebäude in öffentlicher Hand. In Galapagar wurde z.B. ein aufgegebenes Gesundheitszentrum besetzt, um gegen den Abriss des im Eigentum der Gemeinde befindlichen Gebäudes und die geplante Errichtung eines Einkaufszentrums zu protestieren. In aller Regel kamen bei den Besetzungen zwei unterschiedliche Strategien zum Einsatz: In medienwirksamen Besetzungen im Stadtzentrum werden vor allem Gebäude besetzt, um auf die Mechanismen der Spekulation hinzuweisen und sich symbolisch bedeutende Orte anzueignen, während in den Stadtteilen eher die Besetzung von Gebäuden erfolgt, um dort zwangsenteignete Bewohner unterzubringen.

Im Gegensatz zu früheren Hausbesetzungen richtet sich diese selbstverständlich nicht gesetzeskonforme Vorgehensweise vor allem an ganz 'normale', von den wirtschaftlichen und sozialen Verhältnissen sowie den Vorkommnissen der vergangenen Monate empörte Bürger, die sukzessive aktiv wurden und sich radikalisierten. Die Besetzungen lassen sich als Schaffung neuer spaces of citizenship bezeichnen, als Akte des Widerstands gegen ein wirtschaftliches und politisches System, dem es zunehmend an gesellschaftlicher Legitimation mangelt. Dabei 
unterstreicht die Tatsache, dass es sich bei den neuen Aktivisten größtenteils um vormals unpolitische Personen handelt (Martínez/García 2012), wie die im öffentlichen Raum erprobte Politisierung und provokative Überschreitung von Regeln und Gesetzen zur gesellschaftlich legitimierten Neuformulierung dessen führt, was als 'Gemeingut' angesehen wird. Allerdings wird spätestens seit Mitte Dezember auch in Bezug auf die zahlreichen Hausbesetzungen die wesentlich repressivere Politik der neuen konservativen Regierung sichtbar. Die von der Zentralregierung eingesetzte neue Delegada del Gobierno in Madrid, welche die Hoheit über die Nationalpolizei besitzt, verfolgt eine wesentlich härtere Vorgehensweise gegenüber den Mobilisierungen des movimiento 15-M. So wurden jüngere Protestaktionen mit ausufernder Polizeigewalt beantwortet, den Teilnehmern von Versammlungen an der Puerta del Sol wurden Bußgelder angedroht, und es wurde versucht, die Bewegung diskursiv zu delegitimieren und sie als linksextremistische Randerscheinung zu bezeichnen. Auch den Hausbesetzern fällt es in diesem Zusammenhang schwerer, die eroberten spaces of citizenship zu verteidigen - so wurde das Hotel Madrid am 50. Tag seines Bestehens von Hundertschaften der Polizei geräumt. Gleichzeitig findet die Bewegung aber immer wieder neue Ausdrucksformen und Orte zur (Wieder-)Herstellung neuer subversiver Praktiken und acts of citizenship, wie der Slogan 'eine Räumung - eine neue Besetzung' unterstreicht.

\section{Fazit}

Der Bewegung des 15. Mai ist es gelungen, das Machtgefüge zwischen Staat und Gesellschaft neu auszutarieren und die Beziehung zwischen Bevölkerung, Territorium und Regierung mit neuen Inhalten zu füllen. Dies gelang durch die Etablierung subversiver acts of citizenship, durch die gewöhnliche Bürger zu Aktivisten und Subjekten der politischen Auseinandersetzung wurden. Durch die Vergegenständlichung der politischen und sozialen Dimensionen des öffentlichen Raums, die quer zu den hegemonialen Bestrebungen der Vermarktung liegt, gelingt es dem movimiento 15-M neue soziale Beziehungen zu verwirklichen, die zur Ausformulierung dessen führen, was als citizenship bezeichnet werden kann. Hierbei stehen weder die Zugehörigkeit zum Nationalstaat noch die formalen Bürgerrechte im Mittelpunkt, sondern die neuartige Aktivierung und Artikulierung politischer Forderungen. Diese haben sich im Laufe der vergangenen Monate, im Anschluss an die erfolgreiche Rückeroberung des öffentlichen Raums als Ort der Deliberation und Herstellung politischer Öffentlichkeit, sukzessive ausgeweitet. Neue Formen der kollektiven Aktion finden vielfach am Rande der Legalität statt oder überschreiten diese, verfügen aber gleichzeitig über eine hohe 
gesellschaftliche Legitimation. In Bezug auf die angestoßenen Veränderungen steht die Bewegung des 15. Mai nun vor der Herausforderung, die errungenen Positionen zu konsolidieren, zur weiteren Rückeroberung der Stadt durch die neu geschaffenen politischen Subjekte beizutragen, sowie die von den Technologien der Herrschaft befreiten Räume zu einem dichten Netz von Knotenpunkten im Kampf gegen den städtischen Neoliberalismus zu entwickeln. Die in Getafe und Parla, zwei Umlandgemeinden von Madrid, parteiübergreifend getroffenen Entscheidungen, Kommissionen zur Mediation zwischen von Zwangsräumungen bedrohten Familien und Banken einzusetzen und Räumungsklagen während der kommenden drei Monate nicht durchzusetzen, zeigen, dass die Hoffnungen auf andere Formen der städtischen Governance nicht unberechtigt sind.

\section{Literatur}

Abellán, J. (2012, i.Dr.) De la indignación en la red a la acción en la calle. ACME - An International E-Journal for Critical Geographies 11(1).

Arendt, H. (1958): The Human Condition. Chicago.

Amin, A. (2004): Regions unbound. Towards a new politics of place. Geografiska Annaler 86.1: 33-44.

Aradau, C., Huysmans, J.; Squire, V. (2010): Acts of European Citizenship: A Political Sociology of Mobility. Journal of Common Market Studies 48: 945-965.

Barnett, C. (2003): Culture and Democracy. Media, Space and Representation. Edinburgh.

Benhabib, S. (1991): Modelle des öffentlichen Raums. Hannah Arendt, die liberale Tradition und Jürgen Habermas. Soziale Welt 42(2): 147-165.

Brenner, N., Peck, J.; Theodore, N. (2010) Variegated neoliberalization: geographies, modalities, pathways. Global Networks 10.2: 182-222.

Delgado, M. (2011): El espacio público como ideología. Madrid.

Espinar, R. (2012, i.Dr.): El espacio público como escenario de la pugna por la legitimidad democrática: el caso del debate alternativo sobre el Estado de la Nación en el Movimiento 15-M. ACME - An International E-Journal for Critical Geographies 11(1).

Foucault, M. (1990): Tecnologías del yo y otros textos afines. Barcelona.

Garnier, J.-P. (2011): Treinta Objeciones a Horacio Capel. Scripta Nova XV, 353 (1).

Habermas, J. (1990) Der Strukturwandel der Öffentlichkeit. Frankfurt/M.

Howell, P. (1993): Public space and the public sphere: political theory and the historical geography of modernity. Environment and Planning D: Society and Space 11(3): 303-322.

Hughes, N. (2011) 'Young People Took to the Streets and all of a Sudden all of the Political Parties Got Old': The 15M Movement in Spain. Social Movement Studies 10(4): 407-413.

Isin, E. (2008): Theorizing Acts of Citizenship. In: Isin, E.; Nielsen, G. (eds.): Acts of Citizenship. London, 15-43.

- (2009): Citizenship in flux: The figure of the activist citizen. Subjectivity 29: 367-388.

Isin, E.; Wood, P. (1999): Citizenship and Identity. London.

Janoschka, M. (2011): Geografías urbanas en la era del neoliberalismo. Una conceptualización de la resistencia local a través de la participación y la ciudadanía urbana. Investigaciones Geográficas: Boletín del Instituto de Geografia 76: 118-132.

Lees, L.; Slater, T.; Wyly, E. (2008): Gentrification. London. 
Leitner, H.; Sheppard, E.; Sziarto, K.M. (2008). The spatialities of contentious politics. Transactions of the Institute of British Geographers 33(1): 157-172.

Low, M. (2004): Cities as Spaces of Democracy: Complexity, Scale and Governance. Barnett, C.; Low, M. (eds.): Spaces of Democracy. Geographical Perspectives on Citizenship, Participation and Representation. London, 129-146.

Low, S.; Smith, N. (2006): The politics of public space. London.

Marshall, T.H. (1950): Citizenship and social class and other essays. Cambridge.

Martínez, M.; García, A. (2012, i. Dr.): Ocupar las plazas, liberar los edificios. ACME - An International E-Journal for Critical Geographies 11(1).

Massey, D. (2004): Geographies of Responsibility. Geografiska Annaler 86: 5-18.

Ong, A. (2006): Mutations in Citizenship. Theory, Culture \& Society 23: 499-531.

Rose, J. (2001): Contexts of Interpretation: Assessing Immigrant Reception in Richmond, Canada. The Canadian Geographer 45: 474-493.

Sequera, J. (2009): Protestas ciudadanas y movimientos anti-sistémicos en la Comunidad de Madrid. Un estudio de caso: el movimiento por la vivienda digna. Memorias de las Primeras Jornadas de Análisis Político Crítico. Bilbao.

- (2011): Del movimiento vecinal a las movilizaciones por una vivienda digna en Madrid. De la necesidad hecha derecho al derecho hecho necesidad. Nómadas. Revista Critica de Ciencias Sociales y Juridicas.

Shahyar, P. (2011): Vom Tahrir-Platz zur Puerta del Sol. Fünf Thesen über die neuen Protestbewegungen. Analyse \& Kritik - Zeitung für linke Debatte und Praxis 562. Online-Dokument: http://www.akweb.de/ak_s/ak562/26.htm (Zugriff: 28.09.2011).

Smith, N. (1996): The New Urban Frontier: Gentrification and the Revanchist City. London.

Stephens, A. (2010): Citizenship without community: Time, design and the city. Citizenship Studies 14: 31-46.

Tamayo, S. (2006): Espacios de ciudadanía, espacios de conflicto. Sociológica 21.61: 11-40.

Wehrheim, J. (2002): Die überwachte Stadt: Sicherheit, Segregation und Ausgrenzung. Opladen. 